\title{
Exploring the possibilities of transforming Indian cuisine: findings from the Healthy Takeaways project in Lincolnshire (Part I)
}

\author{
G. Middleton ${ }^{1}$ and K. Evans ${ }^{2}$ \\ ${ }^{1}$ College of Social Sciences, University of Lincoln, Brayford Way, Lincoln, Lincolnshire, LN6 7TS, UK and ${ }^{2}$ Social Change \\ Ltd, Sparkhouse Studios, Ropewalk, Lincoln, Lincolnshire, LN6 7DQ, UK
}

Eating Indian food in restaurants and takeaways has become increasingly popular with consumers in the UK ${ }^{(1,2)}$. In recent years there has been a growing emphasis from government and community-based initiatives for major caterers to adopt healthier cooking practices and menus $^{(1)}$. This implementation process is likely to need knowledge of the business culture and understanding of the context faced by industry caterers ${ }^{(1)}$. The Lincolnshire-based Healthy Takeaways project (Part I) aimed to engage with local Indian restaurant and takeaway owners to: 1) understand and observe current methods and decision-making processes when creating cuisine, and 2) inquire on the likelihood of change regarding implementation of healthier cooking and nutritional content options.

To gauge interest, 43 local restaurants and takeaways serving Indian cuisine were contacted for initial consultation and possible participation. In-depth qualitative interviews occurred face-to-face $(n 6)$ and over the telephone $(n 2)$ with takeaway and restaurant staff (owners, managers and head chefs) from different establishments. For eligibility, participants admitted responsibility for making decisions regarding menu, ingredients and cooking techniques. Qualitative data were digitally recorded, transcribed verbatim, supported by field notes and a process of thematic content analysis revealed findings ${ }^{(3)}$. Key informants (a purposive sample) from the restaurants $(n$ ) participated in observations of kitchen-based cooking practices. Observations were made by a trained chef, community nutritionist assistants and the project manager.

Key themes highlighted that restaurants considered the buyer behaviour as important regarding menu setting and utilised an economic sense when purchasing ingredients and food. Staff in the kitchens were trained 'on the job' with limited knowledge on healthy eating. Observational findings suggested that restaurants preferred to cook food in ovens or in large pans, prepared fresh food, including sauces, and used an extensive range of general and specialist Indian food suppliers. Occasionally items were deep fried in vegetable oil. Restaurants used vegetable oil, 'ghee' and artificial colouring extensively, and attributed benefits of flavouring and aesthetic appeal to these items. Furthermore, there was minimal practice of precise measurement and use of specific recipes for allocating amounts of ingredients. The table illustrates views on changing cooking practices and menus in restaurants to create healthier content, and options for the second phase of the project.

\begin{tabular}{ll}
\hline \multicolumn{1}{c}{ Restaurateurs' concerns and opportunities regarding change } \\
\hline Concerns of Indian restaurants & Opportunities for Indian restaurants \\
\hline Reluctance to acknowledge food is unhealthy & Agreement on the importance of promoting healthy food \\
Limited customer-base request healthy meals & Novel business philosophy: making restaurant stand out \\
$\begin{array}{l}\text { Claims of already promoting healthy dishes } \\
\text { Cost of healthier options and remarketing }\end{array}$ & Consideration of reformation of cuisine (with support) \\
promotional materials/menus & Consideration of displaying healthy options (with support) \\
\hline
\end{tabular}

The findings illustrate that Indian restaurants and takeaways are willing to change current practises. Effective interventions are likely to be sensitive to views held by restaurateurs.

1. Leung G (2010) Nutr Bullet 35, 226-234.

2. Mintel (2009) Ethnic Cuisine Mintel Market Intelligence.

3. Harris J, Gleason P, Sheean P et al. (2009) J Am Diet Assoc 109, 80-90. 